\title{
Non-Operating Room Anesthesia and the COVID-19 Patient: Evidence Based Strategies
}

\author{
Ekta Khemani1,2, James Paul1, Saeda S. Nair¹ \\ ${ }^{1}$ Department of Anesthesia, McMaster University, Hamilton, Canada \\ ${ }^{2}$ Department of Anesthesia, Niagara Health System, Saint Catharines, Canada \\ Email: ekta.khemani@niagarahealth.on.ca
}

How to cite this paper: Khemani, E., Paul, J. and Nair, S.S. (2020) Non-Operating Room Anesthesia and the COVID-19 Patient: Evidence Based Strategies. Open Journal of Anesthesiology, 10, 213-223.

https://doi.org/10.4236/ojanes.2020.105019

Received: April 17, 2020

Accepted: May 26, 2020

Published: May 29, 2020

Copyright $\odot 2020$ by author(s) and Scientific Research Publishing Inc. This work is licensed under the Creative Commons Attribution International License (CC BY 4.0).

http://creativecommons.org/licenses/by/4.0/

\section{(c) (i) Open Access}

\begin{abstract}
As non-operating room anesthesia (NORA) vastly increases, so does the complexity of both the patients and the procedures. Unfamiliar environment, remote locations, and limited access to resources are some of the many challenges faced by the anesthesiologist in this environment. Understanding the environmental factors such as the room layout, machine placement and patient positioning needs advanced planning in the COVID-19 patient to determine airway management, intravenous access and overall patient safety. Communication with the proceduralist and healthcare workers (HCW) is of essence as both the procedure and anesthetic requirements may need to be altered as the case progresses. Standard monitoring guidelines should apply to all patients regardless of depth of anesthesia. The COVID-19 patient adds to these challenges. Aerosol generating medical procedures (AGMP's) need to be identified ahead of time in order to ensure the safety of both the staff and patients. This allows for planning and preparation required for transportation of the patient to the room. HCWs may need to be identified and be available to assist with the procedure. Time should be allocated for transportation, briefing, room preparation, personal protective equipment needs of the staff and donning and doffing procedures as per institutional protocol. Communication means with staff outside of the room should be discussed in advance should an emergent situation arise during the procedure. Debriefing should be performed at the end of every procedure to improve patient safety and outcomes.
\end{abstract}

\section{Keywords}

COVID-19, Sedation, Non-Operating Room Anesthesia, Patient Safety

\section{Introduction}

Non-operating Room Anesthesia (NORA) represents a unique challenge to the 
anesthesiologist. According to the American Society of Anesthesiologists (ASA) Closed Claims reports, patients undergoing NORA procedures have a higher risk for morbidity and mortality compared to those undergoing procedures in the operating room (OR) [1]. Suboptimal care and failure to provide safe practice was the leading cause of poor outcomes [1]. However, it has been argued that the work environment for NORA makes it difficult to provide optimal care [2]. NORA procedures are often conducted in remote locations away from supplies, with inadequate workspace, limited equipment, limited assistance, and suboptimal lighting, to name a few of the system factors contributing to the unsafe environment [2].

Moreover, patients undergoing NORA procedures are often medically complex compared to patients undergoing procedures in the OR [3] [4]. Many NORA procedures are added to the procedure list emergently, leaving little time for the anesthesiologist to prepare [3] [4]. A key role of the anesthesiologist is airway management, and this is also impaired by the lack of space and awkward positioning of the patient in these scenarios [4].

Adding to the complexity of NORA procedures, is the stress in managing the suspected or confirmed COVID-19 patient. As the COVID-19 pandemic continues to evolve, there is an anticipation of a surge of COVID-19 patients presenting for NORA procedures. While literature exists for the COVID-19 patient presenting to the OR, there is a paucity of literature for managing the COVID-19 patient undergoing NORA procedures [5]. As such, it behooves the anesthesiologist to develop strategies to limit the transmission from the COVID-19 patient to provide the safest care, for both the patient and the healthcare worker (HCW), under the challenging circumstances a NORA procedure entails.

\section{Pre-Procedural Phase}

\subsection{Screening of Patients}

Paramount to minimizing risk to the anesthesiologist and/or HCW providing care to the patient presenting for a NORA procedure, is effective screening prior to accepting the COVID-19 patient [5] [6]. Moreover, many patients are asymptomatic at the time of their procedure, but later develop symptoms of COVID-19 [5] [6]. Patients undergoing NORA procedures should, therefore, be limited to those requiring urgent or emergent procedures [5].

For urgent or emergent procedures, prior to the NORA procedure, all patients entering the hospital should undergo a series of screening questions. Screening questions [patients vary from institution to institution. An example screening questionnaire from Hamilton Health Sciences (in Hamilton, Ontario, Canada) is listed below:

1) Do you have a fever of $38^{\circ} \mathrm{C}$ or higher or new onset or worsening of cough or difficulty breathing?

2) Do you have any of the following unexplained minor symptoms: runny nose, sore throat, headache, muscle ache, nausea, ansomia (loss of smell) or di- 
arrhea?

3) In the past 14 days, have you returned from travel to any other locations outside of Canada?

4) Has someone in your household, whom you have had close contact with and not worn PPE, tested positive for COVID-19?

To pass this self-screen questionnaire one must answer 'no' to all 4 questions. Patients who do not pass this self-screen will undergo testing. In many institutions, it is often not possible to test all patients undergoing NORA procedures due to a limited supply of testing kits. As such, the Centre for Disease Control (CDC) recommends an approach based on priority for testing patients [7]. Hospitalized patients are given priority, followed by those with symptoms of fever, cough, and shortness of breath who are over age sixty-five, underlying health conditions, patients from long term homes, and first responders [7]. All patients with symptoms would follow next, and finally, all other asymptomatic patients would be last on the priority list [7].

Effective communication of the results of a screening questionnaire or test result for the suspected COVID-19 patient in a timely fashion allows for the anesthesiologist to prepare the necessary medications, equipment, and personal protective equipment $(\mathrm{PPE})$ required for the case [5].

\subsection{Transfer of Patients}

COVID-19 patients undergoing NORA procedures should be transferred to the procedure room using the lowest traffic route [6] [8]. Many institutions have created specific COVID-19 transportation policies, and they must be reinforced in order to minimize the transmission of COVID-19 [5]. The COVID-19 patient must wear a surgical or procedural mask at all times [8]. If needed, supplemental oxygen can be delivered with low flows under the surgical mask [8]. Oxygen facemasks are preferred over nasal cannula to reduce dispersion of exhaled air [8]. HCW involved in the transfer of patients should don droplet PPE according to one's own institutional guidelines, and appropriate transfer of care to the HCWs involved in the procedure should be communicated [5].

\subsection{Room Preparation}

A pre-procedure brief of the room preparation should occur with all HCWs, as NORA procedures are often provided in a confined workspace [2]. Ideally, NORA procedures should be conducted in a negative pressure environment for the COVID-19 patient [9]. As this may be unavailable or unreasonable in many institutions, a high efficiency particulate air filter could be added to reduce viral load in the room [9]. When performing aerosol generating medical procedures, air flow exchanges should also be discussed with the engineering team at one's own institution to minimize risk of transmission. [9].

All unnecessary equipment should be removed to prevent contamination [5]. While removing unnecessary equipment for the COVID-19 patient, however, 
minimum standards for the safety of NORA procedures as outlined by the ASA must still be practiced [9]. The layout of the room should be planned including patient positioning, placement of anesthetic machine, and monitors [9]. This is pertinent in the COVID-19 patient to reduce contamination. Disposable plastic sheets should be draped over all necessary equipment such as fluoroscopy or the anesthetic machine [5] [10]. Medications, airway equipment, and invasive monitoring lines (if using) must be prepared in advance [5] [10]. It is advisable that a designated a "runner" be immediately outside the NORA room, and be available to provide equipment or medications that may be needed throughout the procedure [10].

\subsection{PPE}

PPE requirements for the COVID-19 patient is a dynamic landscape. For aerosol generating medical procedures (AGMP), best practice includes a gown, gloves, a face shield, and an N95 mask or powered air purifying respirator [8]. For non-AGMP procedures, droplet precautions including a face shield, gown, gloves and a procedural mask would be considered best practice (see Figure 1) [8]. These recommendations must be aligned with one's own institutional guidelines.

In addition, studies conducted following Severe Acute Respiratory Syndrome, doffing of PPE was the area that was most prone to errors and staff contamination

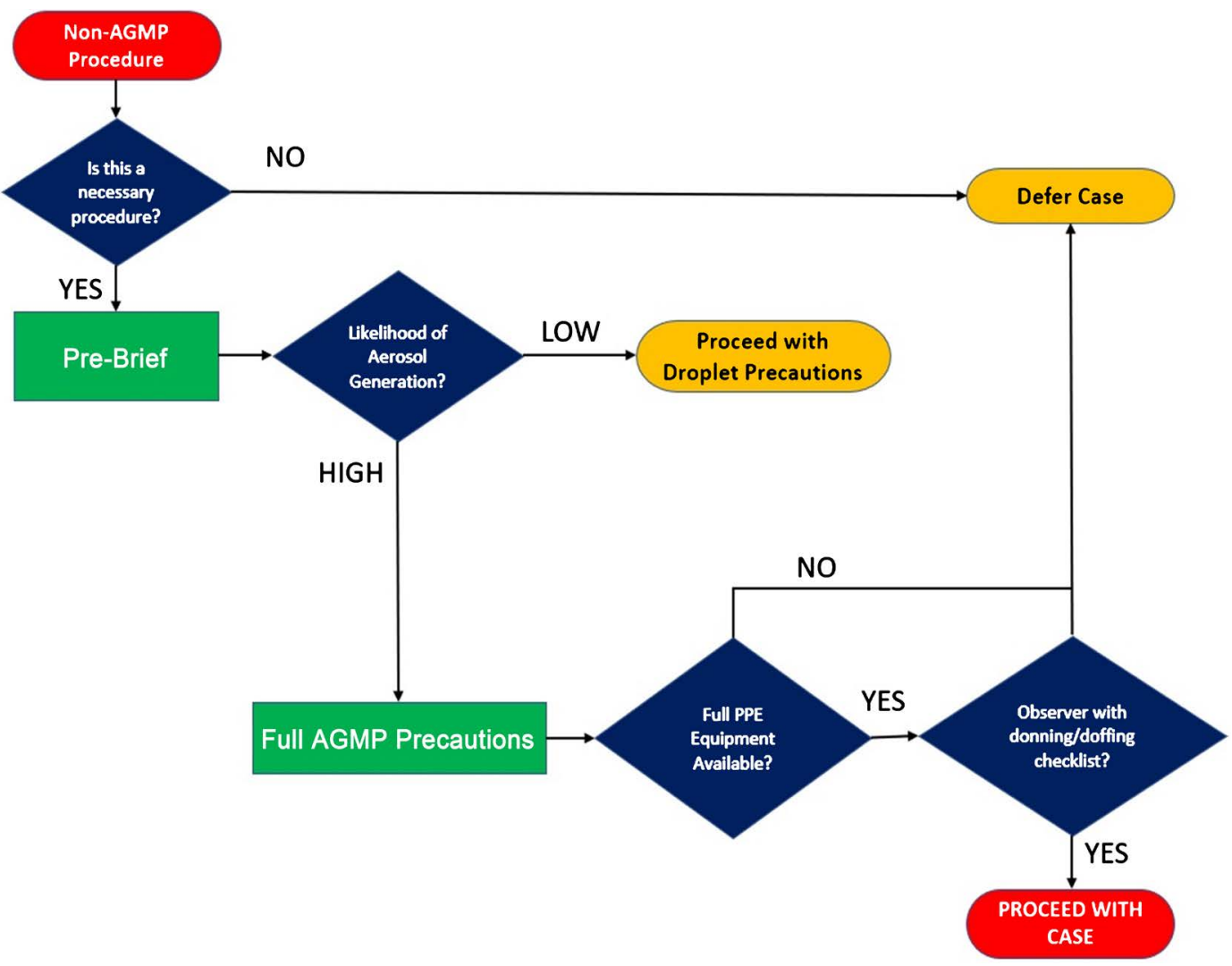

Figure 1. Flowchart for AGMP and Non-AGMP NORA Procedures. 
[11]. In order, to minimize contamination during doffing, checklists of the correct doffing technique should be posted in clear view for all HCWs to observe. It is also recommended that the healthcare team identify and designate a safety officer to observe and read the checklist [11].

\section{Intraprocedural Phase}

Only necessary personnel should be involved in the care of a COVID-19 patient. Many patients receiving NORA procedures are done under sedation and do not have a secured airway [5]. An unplanned need for conversion to a general anesthestic (GA) in a COVID-19 patient is undesirable [8]. If the duration or complexity of the procedure requiring sedation means a high probability of conversion to a GA, it is advisable to start with a GA and use the appropriate PPE [8]. Therefore, strong communication amongst all team members is essential [5] [8].

Appropriate monitoring should be adhered to as per ASA recommendations [12] [13]. The assessment of oxygenation, ventilation and circulation is recommended by the ASA independent of depth, type and location of anesthetic [13]. These recommendations become imperative when dealing with a COVID-19 patient.

The challenges providers face in the non-operating room environment are multifactorial. These include, but are not restricted, to remote location, poor lighting, restricted working environment, and lack of equipment [12] [14]. NORA becomes even more challenging with the COVID-19 patient, adding to the provider's cognitive load. As such, clear and consistent communication is critical to achieving safe outcomes, as patients and provider needs may change during the procedure [8].

Medications given during NORA procedures requiring sedation should be used with caution in COVID-19 patients, as they may have co-existing respiratory compromise from COVID-19 pneumonia [8]. Oxygenation and ventilation should be closely monitored, as inadequate oxygenation and ventilation was responsible for one-third of the NORA malpractice claims cases prior to COVID-19 [1].

When possible, ASA fasting guidelines should be strictly adhered to for the COVID-19 patient undergoing NORA procedures, as aspiration pneumonitis occurred in a higher portion of malpractice claims compared to the OR in the United States [1].

\section{Post Procedural Phase}

To minimize the risk of transmission, the COVID-19 patient should be recovered in the procedure room [15]. Noninfected patients should be removed from waiting areas prior to the transport of the COVID-19 patient [5]. The COVID-19 patient should be transferred to the back to the hospital following a low traffic route [5]. HCW involved in the transport should don PPE [15]. The anesthesiologist should appropriately doff PPE following transport. Since doff- 
ing PPE is when most contamination errors occur, an observer reading a checklist of appropriate doffing techniques should be present at the time of doffing [11]. The procedure room should undergo a terminal clean, including all surfaces [15]. Unused disposable equipment/drugs should be discarded [15]. Reusable equipment should be sterilized as per institutional guidelines [16].

Central the safety of the COVID-19 patient and the anesthesiologist and HCW is debriefing after a NORA procedure. The debriefing should involve all team members involved in the care of the COVID-19 [16]. Debriefing has been shown to improve clinical outcomes in cardiac arrest and reinforces the importance of teamwork and communication amongst team members [16] [17]. Especially as COVID-19 evolves, the need to debrief to improve communication and evaluate systemic issues is critical to patient and staff safety.

\section{Discussion}

While NORA procedures are increasingly in popularity, the management of the COVID-19 patient is challenging. Often, the remote location of NORA procedures and room layout created a series of human factors that can adversely affect patient safety [18]. Confounding this is the suspected COVID-19 patient with the potential for respiratory distress. This underscores the need for meticulous attention and care by the anesthesiologist in caring for the COVID-19 patient.

NORA presents a unique challenge to the anesthesiologist caring for the COVID-19 patient, given the added cognitive load of the unfamiliar environment, in a remote location, with limited access to equipment and drugs. We have summarized our findings into four tables to provide quick aids for the anesthesiologist asked to provide care for NORA procedures (see Appendix 1).

Communication amongst team members is one of the most crucial components to improve patient safety, reduce errors, and to ensure the appropriate precautions are taken. Effective communication is critical prior to accepting a COVID-19 patient for a NORA procedure, throughout the procedure, and at the end of a procedure via a debriefing. Indeed, effective communication is the central premise to many patient safety guidelines [17] [18].

The care of the COVID-19 patient should prioritize safety for both the patient and HCWs involved using the best available evidence-based strategies aligning with institutional protocols. At the same time, patient safety and quality of care should continue to be monitored by using debriefing strategies to identify clinical improvement areas.

\section{Conclusion}

NORA presents several challenges to the anesthesiologist and HCWs caring for the COVID-19 patient, given the added cognitive load of the unfamiliar environment, remote location, with limited access to equipment and medications. To circumvent these issues, the healthcare team must be become very familiar with all the nuances on the procedure room, particularly as it relates to airway man- 
agement to limit transmission of infection. COVID-19 patients should be screened, follow a low traffic route to the procedure room, PPE should be donned and doffed appropriately, and rooms should be stripped of unnecessary equipment [5] [15]. The guiding principles in the management of COVID-19 patients undergoing NORA procedures are open and clear communication throughout the procedure, as needs often change depending on proceduralists needs. The goal of any NORA procedure in the COVID-19 patient is to maximize the care of the infected patient, while minimizing the risk of transmission to healthcare workers and other patients.

\section{Acknowledgements}

We would like to thank Dr. Susan O'Leary and Dr. Donald Duvall for their guidance and support.

\section{Conflicts of Interest}

The authors declare no conflicts of interest regarding the publication of this paper.

\section{References}

[1] Woodward, Z.G., Urman, R.D. and Domino, K.B. (2017) Safety of Non-Operating Room Anesthesia: A Closed Claims Update. Anesthesiology Clinics, 35, 569-581. https://doi.org/10.1016/j.anclin.2017.07.003

[2] Voulgarelis, S. and Scott, J.P. (2017) Monitoring for Nonoperating Room Anesthesia. Anesthesiology Clinics, 35, 591-599. https://doi.org/10.1016/j.anclin.2017.07.004

[3] Metzner, J., Posner, K.L. and Domino, K.B. (2009) The Risk and Safety of Anesthesia at Remote Locations: The US Closed Claims Analysis. Current Opinion in Anesthesiology, 22, 502-508. https://doi.org/10.1097/ACO.0b013e32832dba50

[4] Nagrebetsky, A., Gabriel, R.A., Dutton, R.P. and Urman, R.D. (2017) Growth of Nonoperating Room Anesthesia Care in the United States: A Contemporary Trends Analysis. Anesthesia \& Analgesia, 124, 1261-127. https://doi.org/10.1213/ANE.0000000000001734

[5] Chandy, P.E., Nasir, M.U., Srinivasan, S., Klass, D., Nicolaou, S. and Babu, S.B. (2020) Interventional Radiology and COVID-19: Evidence-Based Measures to Limit Transmission. Diagnostic and Interventional Radiology, 26, 236-240. https://doi.org/10.5152/dir.2020.20166

[6] World Health Organization (2014) Infection Prevention and Control of Epidemic and Pandemic-Prone Acute Respiratory Diseases in Health Care. WHO, Geneva.

[7] (2020)Centers for Disease Control. April 26, 2020. https://www.cdc.gov/coronavirus/2019-ncov/downloads/priority-testing-patients.pdf

[8] Uppal, V., Sondekoppam, R., Lobo, C., Kolli, S., et al. (2020) Practice Recommendations on Neuraxial and Peripheral Nerve Blocks during the COVID-19 Pandemic. ASRA ESRA COVID-19 Guidance for Regional Anesthesia, March 31, 2020.

[9] Alhazzi, W., et al. (2020) Surviving Sepsis Campaign: Guidelines on the Management of Critically Ill Adults with Coronavirus Disease 2019 (COVID-19). Critical Care Medicine, March 27 
[10] Sharma, D., Rasmussen, M., Han, R., Whalin, M., Davis, M., Kofke, W.A., Raghvan, L.V., Raychev, R. and Fraser, J.F. (2020) Anesthetic Management of Endovascular Treatment of Acute Ischemic Stroke During COVID-19 Pandemic: Consensus Statement from Society for Neuroscience in Anesthesiology \& Critical Care (SNACC)_Endorsed by Society of Vascular \& Interventional Neurology (SVIN), Society of NeuroInterventional Surgery (SNIS), Neurocritical Care Society (NCS), and European Society of Minimally Invasive Neurological Therapy (ESMINT). Journal of Neurosurgical Anesthesiology, 32. https://doi.org/10.1097/ANA.0000000000000688

[11] Peng, P.W., Wong, D.T., Bevan, D. and Gardam, M. (2003) Infection Control and Anesthesia: Lessons Learned from the Toronto SARS Outbreak. Canadian Journal of Anesthesia, 50, 989-997. https://doi.org/10.1007/BF03018361

[12] Voulgarelis, S. and Scott, J.P. (2017) Monitoring for Nonoperating Room Anesthesia. Anesthesiology Clinics, 35, 591-599. https://doi.org/10.1016/j.anclin.2017.07.004

[13] American Society of Anesthesiologists Committee on Standards and Practice Parameters (2018) Statement on Nonoperating Room Anesthetizing Locations. October 17,2018

[14] Melloni, C. (2007) Anesthesia and Sedation outside the Operating Room: How to Prevent Risk and Maintain Good Quality. Current Opinion in Anesthesiology, 20, 513-519. https://doi.org/10.1097/ACO.0b013e3282f06ba6

[15] Lie, S., Wong, S., et al. (2020) Practical Considerations for Regional Anesthesia: Lessons Learned from the COVID-19 Pandemic. Canadian Journal of Anesthesia, March 24, 2020. https://doi.org/10.1007/s12630-020-01637-0

[16] Tennenbaum, S.I. and Cesaroli, C.P. (2013) Do Team and Individual Debriefs Enhance Performance? A Meta-Analysis. Human Factors. The Journal of the Human Factors and Ergonomics Society, 55, 231-245.

https://doi.org/10.1177/0018720812448394

[17] Wolfe, H. et al. (2014) Interdisciplinary ICU Cardiac Arrest Debriefing Improves Survival Outcomes. Critical Care Medicine, 42, 1688-1695. https://doi.org/10.1097/CCM.0000000000000327

[18] Walls, J. and Weiss, M. (2019) Safety in Non-Operating Room Anesthesia (NORA). APSF Newsletter, 34, 3-4.

[19] American Society of Anesthesiologists (2013) Statement on Nonoperating Room Anesthetizing Locations. https://www.asahq.org/standards-and-guidelines/statement-on-nonoperating-room -anesthetizing-locations 


\section{Appendix 1}

Issues with non-operating rooms settings and anesthesia [18]

Setting:

- Remote location far from pharmacy and supplies;

- Noisy environments;

- Limited workspace, small procedure room;

- Inadequate lighting;

- Minimal temperature regulation;

- Electrical/magnetic interference;

- Older, possibly unfamiliar equipment;

- Lack of skilled anesthesia support staff;

- Limited patient access during procedures;

- Inadequate power supply;

- Radiation safety.

Anesthesia

- Supply of equipment;

- Appropriate monitoring devices;

- Inadequate support staff;

- Patient-related illness;

- More cases after normal working hours;

- Increased percentage of "emergency" procedures.

Issues with COVID-19 cases undergoing NORA [10]

- COVID-19 is a serious viral infection with a high risk of spreading through droplets, aerosols or contaminated surfaces;

- Airborne precautions are universally recommended for aerosol-generating procedures;

- It's often not practical or feasible to test patients for COVID-19 prior to NORA procedures;

- Bag-mask ventilation, intubation, extubation, airway suctioning, and cardiopulmonary resuscitation may result in aerosolization or respiratory secretions.

\section{Management recommendations for NORA cases [19]}

- There should be in each location a reliable source of oxygen adequate for the length of the procedure. There should also be a backup supply. Prior to administering any anesthetic, the anesthesiologist should consider the capabilities, limitations and accessibility of both the primary and backup oxygen sources. Oxygen piped from a central source, meeting applicable codes, is strongly encouraged. The backup system should include the equivalent of at least a full E cylinder;

- There should be in each location an adequate and reliable source of suction. Suction apparatus that meets operating room standards is strongly encouraged; 
- In any location in which inhalation anesthetics are administered, there should be an adequate and reliable system for scavenging waste anesthetic gases;

- There should be in each location: (a) a self-inflating hand resuscitator bag capable of administering at least 90 percent oxygen as a means to deliver positive pressure ventilation; (b) adequate anesthesia drugs, supplies and equipment for the intended anesthesia care; and (c) adequate monitoring equipment to allow adherence to the "Standards for Basic Anesthetic Monitoring." In any location in which inhalation anesthesia is to be administered, there should be an anesthesia machine equivalent in function to that employed in operating rooms and maintained to current operating room standards;

- There should be in each location, sufficient electrical outlets to satisfy anesthesia machine and monitoring equipment requirements, including clearly labeled outlets connected to an emergency power supply. In any anesthetizing location determined by the health care facility to be a "wet location" (e.g., for cystoscopy or arthroscopy or a birthing room in labor and delivery), either isolated electric power or electric circuits with ground fault circuit interrupters should be provided;

- There should be in each location, provision for adequate illumination of the patient, anesthesia machine (when present) and monitoring equipment. In addition, a form of battery-powered illumination other than a laryngoscope should be immediately available;

- There should be in each location, sufficient space to accommodate necessary equipment and personnel and to allow expeditious access to the patient, anesthesia machine (when present) and monitoring equipment;

- There should be immediately available in each location, an emergency cart with a defibrillator, emergency drugs and other equipment adequate to provide cardiopulmonary resuscitation;

- There should be in each location adequate staff trained to support the anesthesiologist. There should be immediately available in each location, a reliable means of two-way communication to request assistance;

- For each location, all applicable building and safety codes and facility standards, where they exist, should be observed;

- Appropriate postanesthesia management should be provided (see Standards for Postanesthesia Care). In addition to the anesthesiologist, adequate numbers of trained staff and appropriate equipment should be available to safely transport the patient to a postanesthsia care unit.

\section{Management recommendations for COVID-19 NORA cases}

- Screen patients for COVID-19 on admission to hospital;

- Test patients for COVID-19 if possible;

- Ensure that the procedure location has the necessary equipment to provide safe anesthesia care as per the ASA Guidelines;

- Only include the necessary personnel in the procedure room; 
- Avoid general anesthesia if possible but if conversion to a general anesthetic is likely, it's better to start the procedure that way instead of converting to that later;

- Ensure all personnel don the appropriate PPE;

- Recover the patient in the procedure room to avoid risk of transmission;

- Transport the patient back to their care ward via a low-traffic route;

- Debrief with the healthcare team members at the conclusion of the case. 